EGU21-724, updated on 18 Jan 2022

https://doi.org/10.5194/egusphere-egu21-724

EGU General Assembly 2021

(c) Author(s) 2022. This work is distributed under

the Creative Commons Attribution 4.0 License.

\title{
The Art of Identifying Equatorial Waves
}

Peter Knippertz ${ }^{1}$, Juliana Dias ${ }^{2}$, Andreas H. Fink ${ }^{1}$, Maria Gehne ${ }^{2}$, George Kiladis ${ }^{2}$, Kazuyoshi Kikuchi $^{3}$, John Methven ${ }^{4}$, Athul Rasheeda Satheesh ${ }^{1}$, Paul E. Roundy ${ }^{5}$, Andreas Schlueter ${ }^{6}$, Matthew C. Wheeler ${ }^{7}$, Steven J. Woolnough ${ }^{4}$, Gui-Ying Yang ${ }^{4}$, and Nedjeljka Žagar ${ }^{8}$ ${ }^{1}$ Institute of Meteorology and Climate Research, Karlsruhe Institute of Technology, Karlsruhe, Germany (peter.knippertz@kit.edu)

${ }^{2}$ NOAA Physical Sciences Laboratory, Boulder, CO, USA

${ }^{3}$ School of Ocean and Earth Science and Technology, University of Hawaii at Manoa, Honolulu, HI, USA

${ }^{4}$ Department of Meteorology, University of Reading, Reading, UK

${ }^{5}$ Department of Atmospheric and Environmental Sciences, University at Albany, NY, USA

${ }^{6}$ Department of Computer Science, Stanford University, Stanford, CA, USA

${ }^{7}$ Bureau of Meteorology, Melbourne, Australia

${ }^{8}$ Meteorological Institute, University of Hamburg, Hamburg, Germany

Equatorial waves are synoptic- to planetary-scale propagating disturbances at low latitudes with frequencies from a few days to several weeks. Here this term includes Kelvin waves, equatorial Rossby waves, mixed-Rossby gravity waves, and inertio-gravity waves, which are closely related to linear wave theory, but also tropical disturbances, African easterly waves, and the intraseasonal Madden-Julian Oscillation. These waves can couple with deep convection, leading to a substantial modulation of rainfall. Recent work has shown that equatorial waves are amongst the dynamical features internal to the troposphere with the longest intrinsic predictability and that some models forecast them with an exploitable level of skill at lead times of up to a few weeks.

A number of methods have been developed to identify and objectively isolate equatorial waves, both in (usually satellite) observations and in model fields. Most of these rely on (or at least refer to) the adiabatic, frictionless linearized primitive equations or shallow water system on the tropical beta plane. Common ingredients to these methods are longitude-time filtering (Fourier or wavelet) and/or projections onto predefined empirical or theoretical dynamical patterns. This paper aims to give an overview of the different methods to isolate the waves and their structures, to discuss underlying assumptions, to provide a systematic comparison, and to reveal advantages and disadvantages of each method. This way this study helps to optimally choose an approach suited to a given problem at hand and to avoid misuse and misinterpretation of the results. 
